\title{
Effect of Doxycycline-Regulated Protein Disulfide Isomerase Expression on the Specific Productivity of Recombinant CHO Cells: Thrombopoietin and Antibody
}

\author{
Chaya Mohan, ${ }^{1}$ Soon Hye Park, ${ }^{1}$ Joo Young Chung, ${ }^{2}$ Gyun Min Lee ${ }^{1}$ \\ ${ }^{1}$ Department of Biological Sciences, Korea Advanced Institute of Science and Technology, \\ 373-1 Kusong-Dong, Yusong-Gu, Daejon 305-701, Korea; telephone: 82-42-869-2618; \\ fax: 82-42-869-2610; e-mail: gyunminlee@kaist.ac.kr \\ ${ }^{2}$ Department of Bioprocess Technology, Bio Polytech College, 315-1 Chaewoon-Ri, \\ Ganggyeong-Eub, Nonsan-Si, Chungnam, Korea \\ Received 21 October 2006; accepted 26 March 2007 \\ Published online 9 April 2007 in Wiley InterScience (www.interscience.wiley.com). DOI 10.1002/bit.21453
}

\begin{abstract}
Protein disulfide isomerase (PDI), one of the ER-resident molecular chaperones, forms and isomerizes disulfide bonds. This study attempts to investigate the effect of PDI expression level on specific productivity $(q)$ of recombinant Chinese hamster ovary $(\mathrm{rCHO})$ cells producing thrombopoietin (TPO) and antibody (Ab). To regulate the PDI expression level, the Tet-Off system was introduced in TPO and $\mathrm{Ab}$ producing $\mathrm{CHO}$ cells, and stable Tet-Off cells (TPO-Tet-Off and Ab-Tet-Off) were screened using the luciferase assay. The doxycycline-regulated PDI expression system in Tet-Off rCHO cells (Tet-TPO-PDI and Tet-AbPDI) was established by the cotransfection of pTRE-PDI and pTK-Hyg expression vector into TPO-Tet-Off and Ab-TetOff cells, respectively. Subsequent screening was done by Western blot analysis of PDI and an enzyme-linked immunosorbent assay of the secreted TPO and antibody. We cultured two Tet-TPO-PDI and two Tet-Ab-PDI clones, and all these clones showed an average of 2.5-fold increase in PDI expression when compared to the basal level. In both these cell lines the PDI expression was tightly controlled by various concentrations of doxycycline. The $q$ of TPO $\left(q_{\mathrm{TPO}}\right)$ was unaffected but that of antibody producing cells was increased by $15-27 \%$ due to the PDI expression level.

Biotechnol. Bioeng. 2007;98: 611-615.

(C) 2007 Wiley Periodicals, Inc.

KEYWORDS: protein disulfide isomerase (PDI); chinese hamster ovary ( $\mathrm{CHO})$ cells; thrombopoietin (TPO); antibodies
\end{abstract}

\section{Introduction}

Chinese hamster ovary $(\mathrm{CHO})$ cells are one of the most frequently used mammalian hosts for the production of therapeutic glycoproteins (James et al., 1996; Takeuchi et al., 1988). Although gene dosage is one of the key parameters increasing $q$, the post translational process in the endoplasmic reticulum (ER) has been perceived as a ratelimiting step in target protein production (Tuite and Freedman, 1994). In an attempt to overcome the posttranslational bottleneck, ER-resident chaperones, which assist in the later stages of protein synthesis and folding, have been pointed out as a potential target for increasing the $q$ (Chung et al., 2004; Ellis, 1999; Hsu and Betenbaugh, 1997; Hwang et al., 2003; Kleizen and Braakman, 2004; Molinari and Helenius, 2000; Zhang and Kaufman, 2004). To date, various groups have investigated the relationship between $q$ and the activity of chaperones. The effects of chaperones on the specific productivity of recombinant proteins appear to vary among mammalian cell types, chaperones, and target proteins (Borth et al., 2005; Chung et al., 2004; Davis et al., 2000; Hwang et al., 2003).

Protein disulfide isomerase (PDI), one of the well-known ER-resident proteins, is a $58 \mathrm{kDa}$ peptide which increases the rate of formation of native disulfide bonds and catalyzes the rearrangement of non-native disulfide bond formation (Davis et al., 2000; Dorner et al., 1990; Mayer et al., 2000). Studies in vitro on the formation of disulfide bonds in the reoxidation of reduced proteins indicate that the ratedetermining steps are conformational changes accompanied 
by intramolecular thiol-disulfide interchange reactions in which non-native disulfide pairings are replaced by native disulfide pairings (Brockway et al., 1980). As a result, proteins that contain disulfide bonds are often folded slowly in vitro because of the oxidation steps and the requisite correct pairing of cysteine residues. PDI acts not only as an enzyme but also as a molecular chaperone, which was found to associate with misfolded proteins in the ER (Noiva and Lennarz, 1992).

To assess the effect of PDI expression level on the $q$ of rCHO cells, we selected thrombopoietin (TPO) and antibody $(\mathrm{Ab})$ as target proteins. TPO is a highly glycosylated molecule, and posttranslational modification in ER can be a major rate-limiting step in its production. Furthermore, TPO has two disulfide bonds necessary for biological activity in the N-terminal domain. Disruption of these disulfide bonds results in a complete loss of activity (Hoffman et al., 1996). Accordingly, PDI has a possibility to be associated in the posttranslational pathway of TPO in mammalian cells. Antibodies are known to be connected by 16-28 disulfide bonds, depending on the immunoglobulin subtype (Borth et al., 2005). Since disulfide bonds are found to be the crux of activity and conformation of these proteins, it is assumed that PDI is likely to have a positive role in the posttranslational steps of these individual targets and consequently on the $q$ of the $\mathrm{rCHO}$ cells producing these proteins.

To study the effect of PDI expression level on specific TPO productivity $\left(q_{\mathrm{TPO}}\right)$ and specific antibody productivity $\left(q_{\mathrm{Ab}}\right)$ of rCHO cells, we used the Tet-Off System, a tetracycline controlled expression system (Gossen and Bujard, 1992). By employing this type of controlled expression system we exclude the possibility of clonal variability usually encountered in overexpression experiments because the comparison here is made between the same clone at different conditions, and not between two different clones.

\section{Materials and Methods}

\section{Construction of PDI Expression Plasmid}

PDI encoding gene was obtained from the $\mathrm{CHO}$ cDNA pool. The cDNA pool was prepared from mRNA of CHO cells (DUKX-B11, ATCC CRL-9096) using the Marrathon cDNA amplification kit (Clontech, Palo Alto, CA). The PDI cDNA fragment was amplified from the $\mathrm{CHO} \mathrm{cDNA}$ pool by PCR using two primers; PDI-F $=5^{\prime}$ TT GCGGCCGC ATG CTG AGC CGT GCT TTG CT $3^{\prime}$ and PDI-R $=5^{\prime}$ GC GTC GAC CTA CAA TTC GTC CTT TAC AG 3'. The primer sequences were derived from the CHO PDI DNA sequence (Access No. AF364317). The PDI-F primer included a NotI restriction site and the PDI-R primer included a SalI restriction site. The PCR product of PDI cDNA was inserted into the pT7blue $\mathrm{T}$ vector and sequenced by an automated DNA sequencer (ABI prism model 377, Perkin Elmer, Foster City, CA). Double-digestion of pT7blue $\mathrm{T}$ vector by EcoRI and
$\mathrm{XbaI}$ was performed, and the PDI fragment was inserted into a pTRE vector (Clontech), yielding pTRE-PDI.

pTet-Off-Zeo vector was also constructed by inserting a zeocin-resistance gene sequence from pcDNA 3.1(+) vector (Invitrogen, Carlsbad, CA) into a pTet-Off vector for use in Tet-Off-Ab cell line construction.

\section{Generation of Double-Stable Cell Lines}

The rCHO cell lines expressing human TPO (CHO-TPO) and TPO-33-Tet-Off was established in our laboratory as described previously (Hwang et al., 2003).

The double stable Tet-Off-TPO cell line expressing PDI (Tet-TPO-PDI) was established by co-transfecting pTREPDI with pTk-Hyg vector (Clontech) into TPO-33-Tet-Off cells. Upon reaching 90\% confluency, TPO-33-Tet-Off cells were transfected with $3.6 \mu \mathrm{g}$ pTRE-PDI, $0.4 \mu \mathrm{g}$ pTk-Hyg using $10 \mu \mathrm{L}$ Lipofectamine (Invitrogen). To select stably transformed cells, drug selection was carried out for 2 weeks by seeding $1 \times 10^{3}$ cells per well in 96 well tissue-culture plates containing Iscove's modified Dulbeccos' medium (IMDM; Invitrogen) supplemented with 10\% dialyzed fetal bovine serum (dFBS; Invitrogen), $200 \mu \mathrm{g} / \mathrm{mL}$ hygromycin (Sigma), and $0.08 \mu \mathrm{M}$ MTX. Among hygromycin-resistant clones, 22 clones were selected randomly and transferred into 6-well tissue culture plates for expansion. For a negative control, TPO-33-Tet-Off cells transfected with a null pTRE vector (Tet-TPO-Null) were also established.

The rCHO cell line expressing recombinant antibody (CHO-Ab) was established in our laboratory by transfection of a vector containing DHFR, heavy chain, and light chain genes into DHFR-deficient CHO cells (DG44) and subsequent DHFR-mediated gene amplification. The CHO-Ab cells were selected at $0.08 \mu \mathrm{M}$ MTX. The double stable Tet-Off-Ab cell line expressing PDI (Tet-Ab-PDI) was also established by following the same procedure described above. For a negative control, Ab-Tet-Off cells transfected with a null pTRE vector (Tet-Ab-Null) was also established.

\section{Culture Maintenance, Medium, and Culture}

The medium for culture maintenance of Tet-TPO-PDI and Tet-TPO-Null cells was IMDM supplemented with $10 \%$ dFBS, $200 \mu \mathrm{g} / \mathrm{mL}$ hygromycin, and $0.08 \mu \mathrm{M}$ MTX. The medium for culture maintenance of Tet-Ab-PDI and TetAb-Null cells was the same as that for Tet-TPO-PDI clones but with the addition of $350 \mu \mathrm{g} / \mathrm{mL}$ zeocin. The cells were maintained as monolayer cultures in $75-\mathrm{cm}^{2} \mathrm{~T}$-flasks (Nunc, Roskilde, Denmark) in a humidified $5 \% \mathrm{CO}_{2}$ incubator at $37^{\circ} \mathrm{C}$. The cells were passed every 3-4 days upon reaching confluency.

Cell cultures were performed with two Tet-TPO-PDI clones (Tet-TPO-PDI-\#4 and Tet-TPO-PDI-\#11) and two Tet-Ab-PDI clones (Tet-Ab-PDI-\#41 and Tet-Ab-PDI-\#57). For cell culture, exponentially growing cells were inoculated into 6-well tissue culture plates with $3 \mathrm{~mL}$ of the 
maintenance medium containing $1 \mu \mathrm{g} / \mathrm{mL}$ doxycycline. The initial cell concentration was approximately $0.7 \times 10^{5}$ cells/ $\mathrm{mL}$. After 2 days of cultivation in a humidified $5 \% \mathrm{CO}_{2}$ incubator at $37^{\circ} \mathrm{C}$, the spent medium was replaced with $3 \mathrm{~mL}$ of the fresh medium with various concentrations of doxycycline. Culture plates were sacrificed periodically to determine cell concentration and viability. Culture supernatants were aliquoted and kept at $-70^{\circ} \mathrm{C}$ for further analysis.

\section{Western Blot Analysis}

To determine the intracellular PDI expression level, Western blot analysis was performed as described previously (Hwang et al., 2003). PDI was probed with a mouse PDI monoclonal antibody (Stressgen, Victoria, Canada) as a primary antibody and a horseradish peroxidase (HRP)-conjugated anti-mouse IgG goat polyclonal antibody (Upstate, Lake Placid, NY) as a secondary antibody.

\section{Cell Concentration, Viability, and TPO and Antibody Assays}

Cell concentration was estimated using a hemacytometer. Viable cells were distinguished from dead cells using the trypan blue dye exclusion method. Secreted TPO was quantified using an ELISA according to the protocol provided by R\&D Systems (Minneapolis, MN). Secreted antibody concentration was quantified by an ELISA as described previously (Kim et al., 1996, 1998; Yoon et al., 1998).

\section{Evaluation of Specific Growth Rate, $q_{\mathrm{TPO}}$, and $q_{\mathrm{Ab}}$}

The specific growth rate $(\mu)$ was based on the data collected during the exponential growth phase and was evaluated as described earlier (Lee et al., 1991). The $q_{\mathrm{TPO}}$ and $q_{\mathrm{Ab}}$ was based on the data collected during the 2-day culture after the medium exchange with doxycycline, with sampling being done every $12 \mathrm{~h}$. When the TPO and antibody concentrations are plotted against the time integral values of the growth curve, the slope represents $q_{\mathrm{TPO}}$ and $q_{\mathrm{Ab}}$, respectively.

\section{Results}

\section{Cultures of Tet-TPO-PDI Clones With Regulated PDI Expression}

To analyze the effect of PDI expression on cell growth and TPO production, Tet-TPO-PDI (clone \#4 and \#11) were cultivated initially in the maintenance medium containing $1 \mu \mathrm{g} / \mathrm{mL}$ doxycycline. After $24 \mathrm{~h}$, the spent media was
Table I. The $\mu$ and $q_{\mathrm{p}}$ in culture of Tet-TPO-PDI and Tet-Ab-PDI clones at various doxycycline concentrations.

\begin{tabular}{lccc}
\hline Clone \# & Dox $(\mu \mathrm{g} / \mathrm{mL})$ & $\mu(/$ day $)$ & $q_{\mathrm{p}}\left(\mu \mathrm{g} / 10^{6} \text { cells/day }\right)^{\mathrm{a}}$ \\
\hline Tet-TPO-PDI-\#4 & 0 & $0.36 \pm 0.06^{\mathrm{b}}$ & $1.49 \pm 0.11$ \\
& 2 & $0.31 \pm 0.02$ & $1.76 \pm 0.05$ \\
Tet-TPO-PDI-\#11 & 0 & $0.45 \pm 0.02$ & $0.70 \pm 0.12$ \\
& 2 & $0.43 \pm 0.02$ & $0.62 \pm 0.14$ \\
Tet-Ab-PDI-\#41 & 0 & $0.72 \pm 0.06$ & $3.86 \pm 0.29$ \\
& 2 & $0.79 \pm 0.08$ & $2.82 \pm 0.08$ \\
Tet-Ab-PDI-\#57 & 0 & $0.31 \pm 0.07$ & $1.20 \pm 0.01$ \\
& 2 & $0.26 \pm 0.06$ & $1.02 \pm 0.05$ \\
\hline
\end{tabular}

${ }^{\text {aThe }} q_{\mathrm{p}}$ was based on the data collected for 2 days of cultivation after medium exchange, with sampling being done every $12 \mathrm{~h}$.

${ }^{\mathrm{b}}$ Means $\pm \mathrm{SD}$; Standard deviation between the triplicates.

exchanged with fresh media containing four different concentrations of doxycycline $(0,0.01,0.1$, and $2 \mu \mathrm{g} / \mathrm{mL})$.

For all the clones tested, the presence of doxycycline up to $2 \mu \mathrm{g} / \mathrm{mL}$ did not significantly influence the cell growth. The $q_{\text {TPO }}$ of Tet-TPO-PDI-\#11 was $0.70 \pm 0.12 \mu \mathrm{g} / 10^{6}$ cells/day at $0 \mu \mathrm{g} / \mathrm{mL}$ doxycycline and $0.62 \pm 0.14 \mu \mathrm{g} / 10^{6}$ cells/day at $2 \mu \mathrm{g} / \mathrm{mL}$ doxycycline. This meant that the $q_{\mathrm{TPO}}$ was unaffected by PDI expression level. The $\mu$ and $q_{\text {TPO }}$ of Tet-TPO-PDI clones cultured at various doxycycline concentrations are summarized in Table I. To confirm whether PDI expression in Tet-TPO-PDI-\#11 was tightly regulated by doxycycline concentrations, Western blot analysis was performed with cells sampled $24 \mathrm{~h}$ after medium exchange. Figure 1A shows that the expression level of PDI was tightly regulated by doxycycline in a dosedependent manner. This result supports that the elevated PDI expression level has no significant effect on the $q_{\mathrm{TPO}}$ of Tet-TPO-PDI-\#11 clone. Like Tet-TPO-PDI-\#11 clone, cell growth and TPO production of Tet-TPO-PDI-\#4 clone was not affected significantly by the presence of doxycycline up

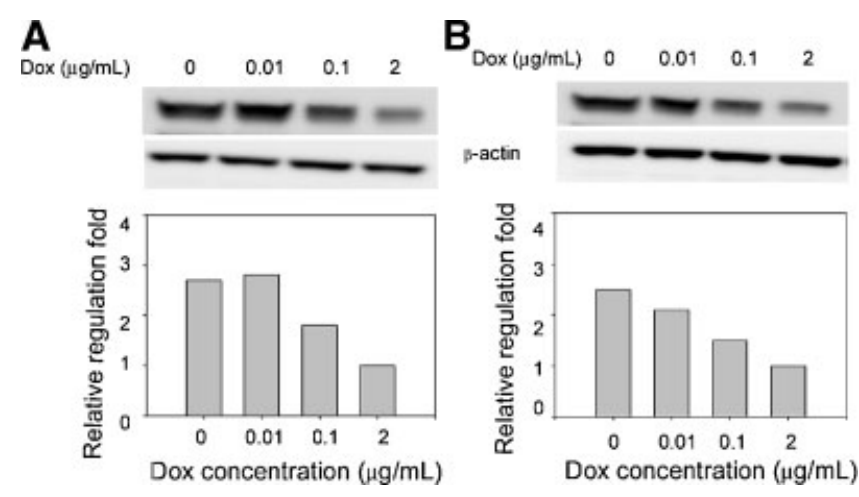

Figure 1. Doxycycline-regulated PDI expression of (A) Tet-TPO-PDI-\#11 clone and (B) Tet-Ab-PDI-\#57 clone. Western blot analysis of clones was performed. Cells were sampled $24 \mathrm{~h}$ after medium exchange. Cells in the absence of doxycycline and in the presence of $0.01,0.1,2 \mu \mathrm{g} / \mathrm{mL}$ doxycycline were loaded at a concentration of $1 \times 10^{5}$ cells $/ 10 \mu \mathrm{L}$. Bar graph indicates the relative PDI expression level at various doxycycline concentrations 
to $2 \mu \mathrm{g} / \mathrm{mL}$, though its PDI expression level was tightly regulated by doxycycline in a dose-dependent manner.

Hence, it can be concluded that, the elevated PDI expression level had no effect on the $q_{\text {TPO }}$ of these TPOproducing rCHO cells.

\section{Cultures of Tet-Ab-PDI Clones With Regulated PDI Expression}

Like the Tet-TPO-PDI clones, Tet-Ab-PDI clones (clone \#41 and \#57) were cultivated in order to analyze the effect of PDI expression on cell growth and antibody production. The elevated PDI expression level did show a positive effect on the specific antibody production rates.

Tet-Ab-PDI-\#57 clone showed only a modest increment in $q_{\mathrm{Ab}}$. The $q_{\mathrm{Ab}}$ was decreased by $2 \mu \mathrm{g} / \mathrm{mL}$ doxycycline to $1.02 \pm 0.05 \mu \mathrm{g} / 10^{6}$ cells/day from $1.20 \pm 0.01 \mu \mathrm{g} / 10^{6}$ cells/ day at $0 \mu \mathrm{g} / \mathrm{mL}$ doxycycline. The $\mu$ and $q_{\mathrm{Ab}}$ of Tet-Ab-PDI clones at various doxycycline concentrations is also summarized in Table I. Western blot analysis showed that the expression level of PDI was tightly regulated by doxycycline in a dose-dependent manner, suggesting that the elevated PDI expression level had less effect on $q_{\mathrm{Ab}}$ of the Tet-Ab-PDI-\#57 clone than expected. (Figure 1B). Tet-AbPDI-\#41 clone had more encouraging results to show. It showed a $q_{\mathrm{Ab}}$ of $2.82 \pm 0.08 \mu \mathrm{g} / 10^{6}$ cells/day at $2 \mu \mathrm{g} / \mathrm{mL}$ doxycycline and $3.86 \pm 0.29 \mu \mathrm{g} / 10^{6}$ cells/day at $0 \mu \mathrm{g} / \mathrm{mL}$ doxycycline. This $27 \%$ increment in the $q_{\mathrm{Ab}}$ was also found to be statistically significant using the Student's $t$-test $(n=3$, $P<0.005)$.

Taken together, it can be concluded that the elevated PDI expression level had a moderately good effect on the $\mathrm{rCHO}$ cells in increasing the $q_{\mathrm{Ab}}$ in the range of $15-27 \%$.

\section{Discussion}

The powerful gene amplification system of $\mathrm{rCHO}$ cells made it possible to obtain high level of gene copies, but secreted protein level was not substantially high compared with its mRNA contents (Chung, 2002). This might be caused by the limitation in translational and/or posttranslational steps, and, if so, increased molecular chaperone levels could relieve the bottleneck in posttranslational modifications in the ER. This hypothesis is supported by the observation that a number of chaperones are expressed in high levels under stressful culture conditions where $q$ is often enhanced (Dorner et al., 1992; Mazzarella et al., 1994).

In this regard, research on engineering the ER chaperones and investigating its effect on the secretion of recombinant proteins has been continuing. To date, the beneficial effect of some chaperones on target protein folding/maturation and on the $q$ of rCHO cells has been reported. Increased levels of ERp57, calnexin and calreticulin were reported to result in enhanced $q$ of TPO in CHO cells (Chung et al., 2004; Hwang et al., 2003). In contrast, the effect of increased level of PDI on $q$ of $\mathrm{rCHO}$ cells is somewhat controversial. The overexpression of PDI decreased the secretion of TNFR:Fc but did not influence the secretion of IL-15 in CHO cells (Davis et al., 2000). It also is also reported that PDI overexpression increased $q_{\mathrm{Ab}}$ of rCHO cells (Borth et al., 2005). However, in these previous reports PDI was overexpressed, and comparison was made between different clones, which bring in the possibility of clonal variability. To investigate the effect of PDI on TPO and antibody producing rCHO cells, we established a tetracyclinecontrolled Tet-Off cell line to control the expression level of the overexpressed PDI. We attempted regulation of the overexpressed PDI rather than its mere overexpression because its expression levels could be a crucial factor for determining their beneficial effect on the specific productivity. Intracellular PDI expression was tightly regulated by various concentrations of doxycycline added. To explore the relationship between the internal PDI expression level and the protein (TPO, antibody) productivity, we cultured the clones with four different concentrations of doxycycline, from $0 \mu \mathrm{g} / \mathrm{mL}$ up to $2 \mu \mathrm{g} / \mathrm{mL}$. In all the four clones investigated, it was observed that in the absence of doxycycline, PDI expression level was increased over 2.5fold compared with that of a basal level seen with $2 \mu \mathrm{g} / \mathrm{mL}$ of doxycycline. However, this enhancement in PDI levels did not entirely translate into $q$ increments. In the case of TPO producing clones, no effect was seen on the $q_{\mathrm{TPO}}$. On the other hand, $q_{\mathrm{Ab}}$ was found to be improved by $15-27 \%$, which was comparable to the previous reported increment of 37\% (Borth et al., 2005).

In this experiment we presumed that PDI would have a decisive role to play in the folding of both, TPO and Ab molecules owing to the presence of important disulfide bonds and hence the overexpression of PDI would eliminate the translational limitation and help in increasing the yield. Stated in simple terms, PDI would help in folding of TPO and $\mathrm{Ab}$ molecules and influence the respective $q_{\mathrm{p}}$ in the rCHO cells. But since, in TPO-producing clones the PDI expression had no effect and in the Ab-producing cells the effect was moderate (15-27\%) it can be stated that PDI has very little effect as a molecular chaperone in the TPO folding pathway and a moderate effect as a molecular chaperone in the antibody folding pathway, so as to not bring about a profound change in the $q$ values.

PDI is known as a catalytic enzyme related in disulfide bond formation of nascent polypeptide chains, as well as a molecular chaperone which assists folding of newly synthesized proteins (Davis et al., 2000; Dorner et al., 1990). It has been seen that PDI's chaperone-like action is not limited to the proteins containing disulfide bonds (Cai et al., 1994), which could mean its chaperone activity may not be related to whether the target protein has disulfide bonds or not. Stated in this context, the mere presence of disulfide bonds in TPO does not qualify it to be acted upon by PDI. Another possible explanation is that basal level expression of PDI is enough for disulfide bond formation and chaperone action in TPO; hence the overexpression did 
not result in any increment in $q$. The interaction between TPO and PDI, however, remains to be clarified.

The effect of overexpression of PDI on the $q_{\mathrm{Ab}}$ has been studied. Overexpression of PDI in combination with BiP reduced the $q_{\mathrm{Ab}}$, whereas overexpression of PDI alone caused an increase of $q_{\mathrm{Ab}}$ (Borth et al., 2005). Our study constructed a well-regulated Tet-Ab-PDI cell line and it did have a modest enhancing influence of about $15-27 \%$ on the $q_{\mathrm{Ab}}$ of the rCHO cell line, which is in agreement with the previously reported increment of 37\% (Borth et al., 2005).

As suggested by an earlier report (Davis et al., 2000), the only slight increment in antibody secretion observed could be due the PDI-associated intracellular retention of the antibody. In another report describing the effect of dexamethasone-induced PDI overexpression on antibody secretion in a murine hybridoma, the authors noted that PDI overexpression had a beneficial effect on the longevity of the PDI-overexpressing cultures but had no effect on secretion rates. In fact they did observe a two-fold lower antibody secretion in the PDI expressing clone with or without induction with dexamethasone (Kitchin and Flickinger, 1995). These reports suggest that the effect of overexpression of PDI on secretion can vary from causing a decrease in the $q$, to having no effect or an enhancing effect.

One can also speculate that since protein folding is a well orchestrated activity with numerous foldases, chaperones, helper proteins and optimum oxidizing environment involved, the overexpression of only PDI may not be high enough to bring about a substantial change in the $q$ of the cells.

In conclusion, it can be said that the results shown here illustrate that PDI has no effect on the $q$ of TPO-producing cell line but certainly has a moderately positive effect on the $q$ of Ab-producing cell line.

This research was supported in part by grants from the Ministry of Commerce, Industry, and Energy, Daejeon city (Bio/RIS program) and the Ministry of Education (Brain Korea 21 Program).

\section{References}

Borth N, Mattanovich D, Kunert R, Katinger H. 2005. Effect of increased expression of protein disulfide isomerase and heavy chain binding protein on antibody secretion in a recombinant $\mathrm{CHO}$ cell line. Biotechnol Prog 21:106-111.

Brockway BE, Forster SJ, Freedman RB. 1980. Protein disulphide-isomerase activity in chick-embryo tissues. Correlation with the biosynthesis of procollagen. Biochem J 191:873-876.

Cai H, Wang CC, Tsou CL. 1994. Chaperone-like activity of protein disulfide isomerase in the refolding of a protein with no disulfide bonds. J Biol Chem 269:24550-24552.

Chung JY. 2002. Development of recombinant Chinese hamster ovary cell lines producing human thrombopoietin analogs. $\mathrm{PhD}$ thesis, Department of Biological Sciences, KAIST, Daejeon, Korea.

Chung JY, Lim SW, Hong YJ, Hwang SO, Lee GM. 2004. Effect of doxycycline-regulated calnexin and calreticulin expression on specific thrombopoietin productivity of recombinant Chinese hamster ovary cells. Biotechnol Bioeng 85:539-546.
Davis R, Schooley K, Rasmussen B, Thomas J, Reddy P. 2000. Effect of PDI overexpression on recombinant protein secretion in $\mathrm{CHO}$ cells. Biotechnol Prog 16:736-743.

Dorner AJ, Wasley LC, Raney P, Haugejorden S, Green M, Kaufman RJ. 1990. The stress response in Chinese hamster ovary cells. Regulation of ERp72 and protein disulfide isomerase expression and secretion. J Biol Chem 265:22029-22034.

Dorner AJ, Wasley LC, Kaufman RJ. 1992. Overexpression of GRP78 mitigates stress induction of glucose regulated proteins and blocks secretion of selective proteins in Chinese hamster ovary cells. EMBO J 11:1563-1571.

Ellis RJ. 1999. Molecular chaperones: Pathways and networks. Curr Biol 9:R137-R139.

Gossen M, Bujard H. 1992. Tight control of gene expression in mammalian cells by tetracycline-responsive promoters. Proc Natl Acad Sci USA 89:5547-5551.

Hoffman RC, Andersen H, Walker K, Krakover JD, Patel S, Stamm MR, Osborn SG. 1996. Peptide, disulfide, and glycosylation mapping of recombinant human thrombopoietin from ser1 to Arg246. Biochemistry 35:14849-14861.

Hsu TA, Betenbaugh MJ. 1997. Coexpression of molecular chaperone BiP improves immunoglobulin solubility and IgG secretion from Trichoplusia ni insect cells. Biotechnol Prog 13:96-104.

Hwang SO, Chung JY, Lee GM. 2003. Effect of doxycycline-regulated ERp57 expression on specific thrombopoietin productivity of recombinant CHO cells. Biotechnol Prog 19:179-184.

James DC, Goldman MH, Hoare M, Jenkins N, Oliver RW, Green BN, Freedman RB. 1996. Posttranslational processing of recombinant human interferon-gamma in animal expression systems. Protein Sci 5:331-340.

Kim JH, Bae SW, Hong HJ, Lee GM. 1996. Decreased chimeric antibody productivity of $\mathrm{KR} 12 \mathrm{H}-1$ transfectoma during long-term culture results from decreased antibody gene copy number. Biotechnol Bioeng 51:479-487.

Kitchin K, Flickinger MC. 1995. Alteration of hybridoma viability and antibody secretion in transfectomas with inducible overexpression of protein disulfide isomerase. Biotechnol Prog 11:565-574.

Kleizen B, Braakman I. 2004. Protein folding and quality control in the endoplasmic reticulum. Curr Opin Cell Biol 16:343-349.

Lee GM, Varma A, Palsson BO. 1991. Production of monoclonal antibody using free-suspended and immobilized hybridoma cells: Effect of serum. Biotechnol Bioeng 38:821-830.

Mayer M, Kies U, Kammermeier R, Buchner J. 2000. BiP and PDI cooperate in the oxidative folding of antibodies in vitro. J Biol Chem 275:2942129425.

Mazzarella RA, Marcus N, Haugejorden SM, Balcarek JM, Baldassare JJ, Roy B, Li LJ, Lee AS, Green M. 1994. Erp61 is GRP58, a stress-inducible luminal endoplasmic reticulum protein, but is devoid of phosphatidylinositide-specific phospholipase $\mathrm{C}$ activity. Arch Biochem Biophys 308:454-460.

Molinari M, Helenius A. 2000. Chaperone selection during glycoprotein translocation into the endoplasmic reticulum. Science 288:331-333.

Noiva R, Lennarz WJ. 1992. Protein disulfide isomerase. A multifunctional protein resident in the lumen of the endoplasmic reticulum. J Biol Chem 267:3553-3556.

Takeuchi M, Takasaki S, Miyazaki H, Kato T, Hoshi S, Kochibe N, Kobata A. 1988. Comparative study of the asparagine-linked sugar chains of human erythropoietins purified from urine and the culture medium of recombinant Chinese hamster ovary cells. J Biol Chem 263:3657-3663.

Tuite MF, Freedman RB. 1994. Improving secretion of recombinant proteins from yeast and mammalian cells. Rational or empirical design? Trends Biotechnol 12:432-434.

Yoon SK, Ahn YH, Kwon IC, Han K, Song JY. 1998. Influence of reducing agents on the secretion rate of recombinant erythropoietin from $\mathrm{CHO}$ cells. Biotechnol Lett 20:101-104.

Zhang K, Kaufman RJ. 2004. Signaling the unfolded protein response from the endoplasmic reticulum. J Biol Chem 279:25935-25938. 